\title{
Estudo epidemiológico do trauma ortopédico em um serviço público de emergência
}

\author{
Epidemiologic of orthopedic trauma study in a \\ public emergency
}

\author{
Lúcia de Fátima da Silva Santos ${ }^{1}$, Juliany Marques Abreu da Fonseca ${ }^{1}$, \\ Bruna Lorena Soares Cavalcante ${ }^{2}$, Cremilda Monteiro Lima ${ }^{1}$
}

\begin{abstract}
Resumo
Introdução: O trauma vem se destacando como grave problema de saúde pública, pois compromete a funcionalidade do indivíduo e sua participação social e econômica na sociedade. Método: Estudo retrospectivo mediante informações de 1.390 prontuários. A coleta de dados ocorreu de setembro de 2011 a fevereiro de 2012. As variáveis estudadas foram: idade, sexo, mecanismo de trauma, parte do corpo afetada, tratamento realizado, modo de condução, dia da semana em que o atendimento foi realizado, tempo de internação e condição de saída hospitalar. Resultados: Houve predominância de homens, na faixa etária de 18 a 38 anos. Verificou-se maior frequência de trauma ortopédico nos membros inferiores $(23,2 \%)$ em decorrência de acidentes de trânsito $(60,1 \%)$. O tratamento cirúrgico foi o mais realizado $(89,8 \%)$ e a maioria dos pacientes evoluiu para alta $(79,4 \%)$. Domingo foi o dia da semana em que mais vítimas de trauma foram atendidas (18,9\%) e as ambulâncias de municípios do interior foram o principal meio de condução ao hospital (34,1\%). A maioria dos pacientes permaneceu internada pelo período de 1 a 15 dias $(89,4 \%)$. Conclusão: Conclui-se pela necessidade de medidas preventivas do trauma ortopédico associadas à conscientização da população diante da morbidade provocada pelo trauma ortopédico.
\end{abstract}

Palavras-chave: epidemiologia; ortopedia; traumatologia; serviços médicos de emergência.

\begin{abstract}
Introduction: The trauma has emerged as a serious public health problem, because it compromises the individual's functionality as its social and economic participation in society. Method: Retrospective study using information of 1390 records. Data collection occurred from September 2011 to February 2012 and the variables studied were: age, gender, mechanism of injury, the affected body part, treatment performed, conduction mode, day of the week that the service was performed, length of hospital stay and hospital output condition. Results: The results showed a predominance of men in the age group 18-38 years. The orthopedic trauma was found more often in the lower limbs $(23.2 \%)$ due to traffic accidents $(60.1 \%)$. Surgical treatment was the most common $(89.8 \%)$ and the majority of the patients progressed to discharge from the hospital $(79.4 \%)$. Sunday was the day of the week that most trauma victims were admitted (18.9\%) and ambulances from inside the municipalities were the main way to the hospital (34.1\%). Most patients remained hospitalized for a period of 1 to 15 days (89.4\%). Conclusion: The results confirmed the need for preventive measures of orthopedic trauma associated with the awareness of the population on the morbidity caused by orthopedic trauma.

Keywords: epidemiology; orthopedics; traumatology; emergency medical services.
\end{abstract}

${ }^{1}$ Universidade Federal do Piauí (UFPI) - Teresina (PI), Brasil.

${ }^{2}$ Instituto de Ensino e Pesquisa, Hospital Sírio Libanês - São Paulo (SP), Brasil.

Trabalho realizado no Hospital de Urgências de Teresina Professor Zenon Rocha (HUT) - Teresina (PI), Brasil.

Endereço para correspondência: Lúcia de Fátima da Silva Santos - Centro de Ciências da Saúde (CCS), Universidade Federal do Piauí, Avenida Frei Serafim, 2280

- Centro - CEP: 64001-020 - Teresina (PI), Brasil -Email: lucia3584@hotmail.com

Fonte de financiamento: nenhuma.

Conflito de interesses: nada a declarar.

Cad. Saúde Colet., 2016, Rio de Janeiro, 24 (4): 397-403 


\section{INTRODUÇÃO}

O trauma é um agravo à saúde, definido como um evento nocivo caracterizado por alterações estruturais ou pelo desequilíbrio fisiológico do organismo resultante da troca de energia entre os tecidos e o meio ${ }^{1,2}$. É considerado uma morbidade que compromete a funcionalidade do indivíduo e sua participação social e econômica na sociedade ${ }^{3}$.

Por ano cerca de 60 milhões de pessoas no mundo sofrem algum tipo de traumatismo, contribuindo com uma em cada seis internações hospitalares ${ }^{2}$. Considerando essa realidade e a dimensão dos números envolvidos, o trauma vem ocupando espaço de destaque nas estatísticas de diagnósticos e internações hospitalares, caracterizando-se como a pandemia da vida moderna e um grave problema de saúde pública, uma vez que atingiu o primeiro lugar como etiologia de morbimortalidade na população de 0 a 39 anos de idade ${ }^{4-7}$.

Assim, conhecer a epidemiologia do trauma se torna essencial para descrever a morbidade, a incapacidade e as limitações das vítimas, bem como definir o alvo mais importante para a prevenção a partir da gravidade das lesões ${ }^{8}$.

Diante do exposto, o presente estudo teve como objetivo descrever o perfil das vítimas de trauma ortopédico internadas em um hospital público de Teresina, no Estado do Piauí.

\section{MÉTODOS}

Trata-se de um estudo epidemiológico, retrospectivo, com abordagem quantitativa, desenvolvido no Hospital de Urgências de Teresina Professor Zenon Rocha (HUT).

Os dados foram coletados mediante análise de 1.566 prontuários no período de setembro de 2011 a fevereiro de 2012. As variáveis estudadas foram: idade, sexo, mecanismo de trauma, parte do corpo afetada, tratamento realizado, condição de saída hospitalar, modo de condução, dia da semana em que o atendimento foi realizado e tempo de internação.

No estudo, incluíram-se pacientes maiores de 18 anos, internados na clínica ortopédica do hospital, com diagnóstico de lesão traumática ortopédica comprovada por laudos de exames complementares anexados aos prontuários ou nestes descritos.

Neste estudo, definiu-se trauma conforme a Classificação Internacional de Doenças, $10^{a}$ Revisão (CID-10) ${ }^{9}$. Consideraram-se as CIDs de S00 a T14 pertencentes ao capítulo XIX, "Lesões, envenenamento e algumas outras consequências de causas externas", uma vez que se relacionam às condições traumáticas. O mecanismo de trauma foi classificado de acordo com critérios preconizados pela Organização Mundial de Saúde (OMS) expresso na CID- $10^{10}$.

O banco de dados foi construído no programa Microsoft Office Excel (Microsoft ${ }^{\circledR}$ ) 2007 por meio de dupla digitação a fim de evitar inconsistências. Posteriormente, exportaram-se os dados para planilhas do Statistical Package for the Social Sciences
(SPSS), versão 18.0, para análise estatística, a qual constou de descrição das frequências das variáveis e associação entre as variáveis nominais (mecanismos de trauma, tratamento realizado e condição de saída hospitalar), sem utilização de fator de ponderação. Para tanto, foram aplicados os testes de qui-quadrado de associação, complementado pelo teste exato de Fisher nos casos em que a frequência esperada de observação fosse menor do que cinco. Nas análises de associação, o mecanismo de trauma foi considerado como variável dependente. Considerou-se o nível de significância de $5 \%(\mathrm{p}<0,05)$ para rejeição da hipótese de igualdade entre os grupos estudados.

Em cumprimento à Resolução no 466/2012, do Conselho Nacional de Saúde do Ministério da Saúde, previamente à realização do estudo, o projeto foi submetido à avaliação do Comitê de Ética em Pesquisa da Universidade Estadual do Piauí e aprovado conforme Parecer no 63055, CAAE nº 02454412.5.0000.5209.

\section{RESULTADOS}

Foram analisados 1.566 prontuários pertencentes a pacientes internados na clínica ortopédica do HUT no período de $1^{\circ}$ de setembro de 2011 a 29 de fevereiro de 2012. Destes, 1.390 foram incluídos no estudo, e 176, excluídos por não obedecerem aos critérios de inclusão.

A análise quanto ao perfil epidemiológico evidenciou que a maior parte das vítimas de trauma ortopédico internadas no HUT, no período analisado, constitui-se por homens (81\%) e jovens entre 18 e 38 anos $(61,9 \%)$ (Tabela 1$)$.

Os dados da Tabela 2 demonstram que os acidentes de trânsito foram os mecanismos de trauma mais frequentes $(60,2 \%)$. Os membros inferiores foram os mais afetados, com destaque para o segmento joelho/perna (23,2\%), seguidos pelo acometimento de múltiplas regiões $(23,2 \%)$. O tratamento cirúrgico foi o mais realizado $(89,8 \%)$ em todos os mecanismos de trauma, com maior predominância entre as vítimas de acidente de moto (45,8\%). A maioria dos pacientes evoluiu para alta, com melhora do quadro $(79,4 \%)$. Em toda a população estudada houve registro de apenas três óbitos $(0,2 \%)$. As ambulâncias de municípios do interior foram o principal meio de condução dos

Tabela 1. Perfil epidemiológico dos pacientes vítimas de trauma ortopédico atendidos no Hospital de Urgências de Teresina Professor Zenon Rocha (HUT), no período de setembro de 2011 a fevereiro de 2012, em Teresina, no Piauí

\begin{tabular}{lcc}
\multicolumn{1}{c}{ Variável } & n & \% \\
$\begin{array}{l}\text { Sexo } \\
\text { Masculino }\end{array}$ & 1126 & 81,0 \\
$\quad$ Feminino & 264 & 19,0 \\
Idade & & \\
$18-38$ & 861 & 61,9 \\
$39-59$ & 361 & 26,0 \\
$60-80$ & 134 & 9,6 \\
$81-100$ & 34 & 2,4 \\
\hline
\end{tabular}


Tabela 2. Caracterização da amostra quanto às variáveis relativas ao trauma, no período de setembro de 2011 a fevereiro de 2012, em Teresina, no Piauí Mecanismo de trauma

Acidentes de trânsito/carro

Acidentes de trânsito/motociclista

Acidentes de trânsito/pedestre/atropelamento

Acidentes de trânsito envolvendo animal

Prática esportiva

Quedas

Preensão/esmagamento

Agressão física

Ferimento por perfurocortante

Tentativa de suicídio

Outros

Parte do corpo afetada

Cabeça e pescoço

Tórax

Abdome/lombar/dorso/pelve

Ombro/braço

Cotovelo/antebraço

Punho/mão

Quadril/coxa

Joelho/perna

Tornozelo/pé

Múltiplas regiões

Tratamento realizado

Conservador

Cirúrgico

Não especificado

Conservador e cirúrgico

Condição de saída hospitalar

Alta

Alta a pedido

Alta por evasão

Transferência

Óbito

Modo de condução

Veículo próprio ou de terceiros

Ambulância do serviço de atendimento móvel de urgência (SAMU)

Ambulância qualquer (na cidade de Teresina)

Ambulância de unidades do interior

Veículo da polícia rodoviária federal

Veículo da polícia militar

Ônibus ou a pé

Ambulância do corpo de bombeiros

Não informado

Dias da semana

Domingo

Segunda-feira

Terça-feira

Quarta-feira

Quinta-feira

Sexta-feira

Sábado

Tempo de internação (dias)

1-15

16-30

$31-60$

61-80

81-101 n

$\%$

4,3

$695 \quad 50$

$69-5$

$12 \quad 0,9$

$1 \quad 0,1$

$249 \quad 17,9$

$1 \quad 0,1$

$68 \quad 4,9$

1128,1

$1 \quad 0,1$

1228,8

$9 \quad 0,6$

$1 \quad 0,1$

$2 \quad 0,1$

$138 \quad 9,9$

$157 \quad 11,3$

$144 \quad 10,4$

$147 \quad 10,6$

$323 \quad 23,2$

$146 \quad 10,5$

$322 \quad 23,2$

$81 \quad 5,8$

$1243 \quad 89,4$

$61 \quad 4,4$

$5 \quad 0,4$

$1104 \quad 79,4$

$17 \quad 1,2$

$22 \quad 1,6$

$244 \quad 17,6$

$3 \quad 0,2$

$382 \quad 27,5$

$407 \quad 29,3$

$71 \quad 5,1$

$474 \quad 34,1$

20,1

$9 \quad 0,6$

$31 \quad 2,2$

10,1

$13 \quad 0,9$

$263 \quad 18,9$

$211 \quad 15,2$

$199 \quad 14,3$

$195 \quad 14,0$

$192 \quad 13,8$

$159 \quad 11,4$

$171 \quad 12,3$

$1242 \quad 89,4$

$104 \quad 7,4$

$37 \quad 2,7$

$6 \quad 0,4$

$1 \quad 0,1$ 
pacientes ao hospital (34,1\%). Durante o período estudado, o domingo foi o dia da semana em que houve maior número de atendimento de pacientes vítimas de trauma (18,9\%). A maioria dos pacientes permaneceu internada no hospital por um período de 1 a 15 dias $(89,4 \%)$.

Conforme é apresentado na Tabela 3, a abordagem cirúrgica foi a conduta adotada no tratamento de $91,6 \%$ dos pacientes internados por acidente de moto e de $94,1 \%$ dos pacientes internados vítimas de trauma por agressão física.

A condição de saída hospitalar apontou elevado percentual de alta $(79,4 \%)$, seguido das transferências $(17,5 \%)$. No período do estudo, registraram-se apenas três óbitos $(0,2 \%)$, todos em vítimas de acidente de moto (Tabela 4).

\section{DISCUSSÃO}

O HUT foi fundado em 1941 e localiza-se na zona Centro-Sul do município de Teresina. Trata-se de um serviço público de referência no atendimento de urgência e presta atendimento a pacientes de todas as faixas etárias, do próprio Estado e de alguns Estados vizinhos, como Maranhão, Pará e Tocantins ${ }^{11}$.

O perfil clínico-epidemiológico dos pacientes internados na clínica traumato-ortopédica do HUT foi formado por indivíduos predominantemente do sexo masculino, jovens, vítimas de trauma por acidentes de trânsito, com destaque para aqueles envolvidos com motocicletas e atropelamentos.

Atribui-se ao predomínio de vítimas jovens e do gênero masculino ${ }^{12,13}$ a exposição dessa população a fatores de risco,

Tabela 3. Distribuição das vítimas de trauma ortopédico segundo o tratamento realizado e mecanismo de trauma, no período de setembro de 2011 a fevereiro de 2012, em Teresina, no Piauí

\begin{tabular}{|c|c|c|c|c|c|}
\hline \multirow[b]{2}{*}{ Mecanismo de trauma } & \multicolumn{5}{|c|}{ Tratamento realizado } \\
\hline & Conservador & Cirúrgico & $\begin{array}{c}\text { Não } \\
\text { especificado }\end{array}$ & $\begin{array}{l}\text { Conservador e } \\
\text { cirúrgico }\end{array}$ & Total \\
\hline Acidentes de trânsito/carro & 2 & 57 & 0 & 1 & 60 \\
\hline Acidentes de trânsito/motociclista & 33 & 637 & 24 & 1 & 695 \\
\hline Acidentes de trânsito/pedestre/atropelamento & 3 & 62 & 4 & 0 & 69 \\
\hline Acidentes de trânsito envolvendo animal & 1 & 11 & 0 & 0 & 12 \\
\hline Prática esportiva & 0 & 1 & 0 & 0 & 1 \\
\hline Quedas & 16 & 209 & 23 & 1 & 249 \\
\hline Preensão/esmagamento & 0 & 1 & 0 & 0 & 1 \\
\hline Agressão física & 3 & 64 & 1 & 0 & 68 \\
\hline Ferimento por perfurocortante & 5 & 102 & 3 & 2 & 112 \\
\hline Tentativa de suicídio & 0 & 1 & 0 & 0 & 1 \\
\hline Outros & 18 & 98 & 6 & 0 & 122 \\
\hline Total & 81 & 1243 & 61 & 5 & 1390 \\
\hline
\end{tabular}

p-valor=0,004; Intervalo de confiança de $95 \% ; n=1.390$

Tabela 4. Distribuição das vítimas de trauma ortopédico segundo a condição de saída hospitalar e mecanismo de trauma, no período de setembro de 2011 a fevereiro de 2012, em Teresina, no Piauí

\begin{tabular}{|c|c|c|c|c|c|c|}
\hline \multirow[b]{2}{*}{ Mecanismo de trauma } & \multicolumn{6}{|c|}{ Condição de saída hospitalar } \\
\hline & Alta & $\begin{array}{c}\text { Alta a } \\
\text { pedido }\end{array}$ & $\begin{array}{c}\text { Alta por } \\
\text { evasão }\end{array}$ & Transferência & Óbito & Total \\
\hline Acidentes de trânsito/carro & 47 & 3 & 0 & 10 & 0 & 60 \\
\hline Acidentes de trânsito/motociclista & 525 & 4 & 7 & 156 & 3 & 695 \\
\hline Acidentes de trânsito/pedestre & 57 & 0 & 0 & 12 & 0 & 69 \\
\hline Acidentes de trânsito envolvendo animal & 9 & 1 & 0 & 2 & 0 & 12 \\
\hline Prática esportiva & 0 & 0 & 0 & 1 & 0 & 1 \\
\hline Quedas & 206 & 4 & 4 & 35 & 0 & 249 \\
\hline Preensão/esmagamento & 1 & 0 & 0 & 0 & 0 & 1 \\
\hline Agressão física & 52 & 3 & 4 & 9 & 0 & 68 \\
\hline Ferimento por perfurocortante & 99 & 1 & 3 & 9 & 0 & 112 \\
\hline Tentativa de suicídio & 1 & 0 & 0 & 0 & 0 & 1 \\
\hline Outros & 107 & 1 & 4 & 10 & 0 & 122 \\
\hline Total & 1104 & 17 & 22 & 244 & 3 & 1390 \\
\hline
\end{tabular}

p-valor=0,001; Intervalo de confiança de $95 \% ; n=1.390$ 
como a imaturidade no trânsito, a pouca experiência para dirigir, a motivação e influência do grupo de amigos, o consumo de álcool e drogas ${ }^{14}$, além de ser a faixa etária em que há a influência comportamental representada pela impulsividade e agressividade $e^{6,15}$. Nesse sentido, resultados semelhantes são encontrados em pesquisas nacionais ${ }^{6,8,15-17}$ e internacionais ${ }^{18,19}$.

Neste estudo, as vítimas de acidentes de moto representaram metade da casuística e formaram, com as vítimas de atropelamentos e de acidentes de carro, a grande maioria dos pacientes cirúrgicos.

Acredita-se que o elevado percentual de acidentes envolvendo motociclistas no Estado do Piauí esteja diretamente relacionado ao número de motocicletas circulantes, fenômeno que ocorre na maioria das cidades do país, considerando-se a relação custo/benefício de sua utilização e a aceitação e aprovação da população ${ }^{11}$.

Em geral, motociclistas e pedestres representam as vítimas de ocorrência de trânsito com maior risco de lesões graves e maior necessidade de internamento devido à fragilidade e à falta de proteção do corpo humano diante de um veículo ${ }^{6,8,17,20}$. Além disso, o pedestre possui massa corporal relativamente pequena quando comparada à de um veículo automotor; logo, oferece pouca resistência e absorve a energia do impacto, o que eleva as taxas de morbimortalidade para esse grupo de vítimas ${ }^{17}$.

Observou-se, neste estudo, alta prevalência da abordagem cirúrgica como forma de tratamento em todos os mecanismos de trauma analisados. Esse dado aponta a necessidade de serviços de atendimento preparados para uma abordagem multidisciplinar, considerando que as cirurgias de emergência são frequentes em vítimas de trauma e geralmente realizadas diante de situações clínicas delicadas, tais como trauma tissular maciço e incontrolável, hipovolemia, choque, hipoperfusão, hipóxia tissular, hipotermia e coagulação intravascular disseminada por consumo ${ }^{21}$.

Ademais, o trauma ortopédico exige fixação ortopédica definitiva e precoce, principalmente no paciente politraumatizado, uma vez que essa conduta diminui os índices de morbidade e mortalidade por reduzir as complicações pulmonares e outras decorrentes da permanência prolongada no leito ${ }^{22}$.

Convém salientar também que, em um estudo dos custos de um mês de assistência de emergência realizada em dois hospitais municipais do Rio de Janeiro, verificou-se que, quando considerado o custo total de todas as vítimas da amostra estudada, as cirurgias representaram a variável de custo mais importante ${ }^{23}$. Destaca-se ainda que as internações por traumatismos exigem para o tratamento um gasto médio mais elevado do que aquelas em que o diagnóstico de internação tenha sido uma causa natural ${ }^{24}$.

Apesar de que em nossa realidade não se tenha um cálculo, de forma estruturada, dos custos financeiros envolvidos na atenção às vítimas de trauma ortopédico, mas levando-se em conta a gravidade e o número de vítimas que demandaram por procedimentos cirúrgicos e internações, deduz-se o montante dos recursos gastos pelo Sistema Único de Saúde (SUS) para pagamento desses procedimentos e de como eles constituem gasto significativo para a assistência hospitalar.

Quanto à parte do corpo afetada, estudos analisados ${ }^{25-27}$ mostram que, em vítimas de acidentes, os membros são os segmentos corpóreos mais atingidos ${ }^{25-27}$, visto serem as regiões mais desprotegidas, havendo menor incidência de traumas em regiões da cabeça, tórax e pelve $e^{26,27}$ - dado que corrobora o nosso estudo.

Nos acidentes de trânsito, as extremidades são mais vulneráveis a lesões e são comumente atingidas em decorrência do trauma direto ou após a vítima ser jogada para fora do veículo ${ }^{26}$.

Em motociclistas, os traumas em membros superiores são comuns, uma vez que o equipamento de segurança fornece proteção somente para a cabeça, deixando os membros desprotegidos ${ }^{25,27}$. Nos acidentes automobilísticos, as fraturas de ombro/braço, especificamente as fraturas da diáfise do úmero, podem ser justificadas pelo apoio dos corpos com o braço no painel, muitas vezes para compensar a falta do cinto de segurança no momento da colisão, havendo uma combinação de flexão e grande força de rotação interna na extremidade superior ${ }^{28}$.

Segundo Andrade et al. ${ }^{29}$, a natureza das lesões pode influenciar o tipo de tratamento do paciente, o tempo de internação e as condições de saída hospitalar. Este estudo evidenciou como tempo médio de internação o intervalo de 1 a 15 dias, com um tempo mínimo de 1 dia e máximo de 101 dias, equiparando-se aos resultados encontrados no estudo das características das vítimas de acidentes de motocicletas em hospital de Fortaleza, no Estado do Ceará ${ }^{29}$. Outros estudos também apontam esse tempo como período médio de internação de vítimas de trauma ortopédico ${ }^{30,31}$.

Quando analisados os dias da semana em que ocorreram mais admissões, o domingo e a segunda-feira apresentaram, respectivamente, as maiores frequências. Isso pode ser atribuído à maior incidência de comportamentos perigosos no final de semana, como a ingestão de álcool e o desrespeito às leis de trânsito, resultando em maior número de acidentes nesse período, estendendo a procura por assistência hospitalar também à segunda-feira. Achados semelhantes foram relatados por Ganne $^{32}$ e Vieira et al. ${ }^{16}$.

Em relação à condição de saída hospitalar, este estudo evidenciou grande percentual de alta em contrapartida do registro de óbito. Um estudo que analisou as características e as intercorrências transoperatórias em vítimas de acidentes de trânsito considerou que a realização da cirurgia ortopédica pode atuar como fator de proteção para o óbito nesses pacientes ${ }^{21}$.

As transferências representaram o segundo maior percentual de condição de saída hospitalar neste estudo, o que se justifica por 
se tratar de um hospital de urgência com uma elevada demanda de pacientes. Assim, quando é necessário um segundo tempo cirúrgico ou maiores recursos, parte dos pacientes internados é transferida para outras unidades hospitalares, desocupando os leitos para os quadros agudos ${ }^{13,33}$.

Dentre os meios de condução dos pacientes traumatizados, a ambulância do interior foi o transporte predominantemente utilizado. Desse modo, entende-se que a maioria das vítimas admitidas no serviço estudado é proveniente de cidades do interior do Estado e são levadas ao hospital de referência da capital para receber assistência médica especializada e com melhores recursos.

Diante desses resultados, os índices de morbidade se tornam mais preocupantes, pois o predomínio de adultos jovens nessas ocorrências produz impacto negativo e significativo na economia do Estado, por envolver pessoas em plena idade produtiva ${ }^{14}$.

Esta pesquisa apresentou como limitação, para discussão dos resultados encontrados, a escassez de estudos que tivessem como objetivo conhecer as características epidemiológicas de pacientes vítimas de trauma ortopédico.

\section{CONCLUSÃO}

Os acidentes de trânsito envolvendo motocicletas, carros e pedestres constituem parcela importante das vítimas de trauma ortopédico no município de Teresina. A partir desta investigação, evidencia-se o perfil dos pacientes vítimas de trauma internados na clínica ortopédica do HUT, onde se observa a predominância de indivíduos jovens, principalmente do sexo masculino, a maior parte vítimas de trauma por acidentes de trânsito, provenientes do interior do Estado, submetidos a tratamento cirúrgico, que tiveram como desfecho a alta hospitalar.

A magnitude dos dados apresentados demonstra uma situação alarmante de saúde pública, tendo em vista o risco de morte ou de sequelas graves muitas vezes incapacitantes e/ou permanentes provocadas pelo trauma ortopédico. Diante desse cenário, medidas preventivas do trauma ortopédico associadas à conscientização da população, em especial condutores de motocicletas e de carros, devem ser estimuladas. Ademais, espera-se que os resultados aqui apresentados forneçam subsídios para o estabelecimento de estratégias de organização e de estruturação dos serviços de saúde para o atendimento da população do município.

\section{REFERÊNCIAS}

1. Settervall CHC, Domingues CA, Sousa RMC, Nogueira LS. Mortes evitáveis em vítimas com traumatismos. Rev Saude Publica. 2012;2(46):367-75. PMid:22310649. http://dx.doi.org/10.1590/S0034-89102012005000010.

2. Batista SEA, Baccani JG, Silva RAP, Gualda KPF, Vianna Jr RJA. Análise comparativa entre os mecanismos de trauma, as lesões e o perfil de gravidade das vítimas, em Catanduva - SP. Rev Col Bras Cir. 2006;1(33):6-10. http:// dx.doi.org/10.1590/S0100-69912006000100003.

3. Kfuri Jr M. O trauma ortopédico no Brasil. Rev Bras Ortop. 2011;46(1).

4. Braga Jr MB, Chagas No FA, Porto MA, Barroso TA, Lima ACM, Silva SM, et al. Epidemiologia e grau de satisfação do paciente vítima de trauma músculo-esquelético atendido em hospital de emergência da rede pública brasileira. Acta Ortop Bras. 2005;13(3)

5. Lino Jr W, Segal AB, Carvalho DE, Fregoneze M, Santili C. Análise estatística do trauma ortopédico infanto-juvenil do pronto socorro de ortopedia de uma metrópole tropical. Acta Ortop Bras. 2005;13(4):179-82.

6. Itami LT, Faro ACM, Meneghin P, Leite RCBO, Silveira CT. Adultos com fraturas: das implicações funcionais e cirúrgicas à educação em saúde. Rev Esc Enferm USP. 2009;43(2):1238-43. http://dx.doi.org/10.1590/S008062342009000600016 .

7. Senefonte FRA, Rosa GRPS, Comparin ML, Covre MR, Jafar MB, Andrade FAM, et al. Amputação primária no trauma: perfil de um hospital da região centro-oeste do Brasil. J Vasc Bras. 2012;11(4):269-76. http://dx.doi. org/10.1590/S1677-54492012000400004.

8. Miki N, Martimbianco ALC, Hira LT, Lahoz GL, Fernandes HJA, Reis FB. Profile of trauma victims of motorcycle accidents treated at hospital São Paulo. Acta Ortop Bras. 2014;22(4):219-22. PMid:25246854. http://dx.doi. org/10.1590/1413-78522014220400642.

9. Código Internacional de Doenças. Lesões, envenenamento e algumas outras conseqüências de causas externas. 2015 [citado em 2015 Mar 08]. Disponível em: http://www.cid-10.org/capitulos/19-lesoes-envenenamentoe-algumas-outras-consequencias-de-causas-externas

10. World Health Organization. International Statistical Classification of diseases and related health problems: ICD-10. Geneva: WHO; 2010.

11. Santos AMR, Moura MEB, Nunes BMVT, Leal CFS, Teles JBM. Perfil das vítimas de trauma por acidente de moto atendidas em um serviço público de emergência. Cad Saude Publica. 2008;24(8):1927-38. PMid:18709233. http://dx.doi.org/10.1590/S0102-311X2008000800021.

12. Motoki THC, Carvalho KC, Vendramin FS. Perfil de pacientes vítimas de trauma em membro inferior atendidos pela equipe de cirurgia reparadora do Hospital Metropolitano de Urgência e Emergência. Rev Bras Cir Plást. 2013;28(2):276-81.

13. Carvalho ICC, Saraiva IS. Perfil das vítimas de trauma atendidas pelo serviço de atendimento móvel de urgência. Rev Interd. 2015;8(1).

14. Credo PFD, Felix JVC. Perfil dos pacientes atendidos em um hospital de referência ao trauma em Curitiba: implicações para a enfermagem. Cogitare Enferm. 2012;17(1):126-31. http://dx.doi.org/10.5380/ce.v17i1.26385.

15. Rezende Na DSR, Alves AKS, Leão GM, Araújo AA. Perfil das ocorrências de politrauma em condutores motociclísticos atendidos pelo SAMU de Teresina-PI. Rev Bras Enferm. 2012;65(6):936-41. PMid:23559171. http:// dx.doi.org/10.1590/S0034-71672012000600008.

16. Vieira RCA, Hora EC, Oliveira DV, Vaez AC. Levantamento epidemiológico dos acidentes motociclísticos atendidos em um Centro de Referência ao Trauma de Sergipe. Rev Esc Enferm USP. 2011;45(6):1359-63. PMid:22241193. http://dx.doi.org/10.1590/S0080-62342011000600012. 
17. Castro RRM, Ribeiro NF, Andrade AM, Jaques BD. Profile of patients of orthopedic ward of a public hospital in Salvador-Bahia. Acta Ortop Bras. 2013;21(4):191-4. PMid:24453666. http://dx.doi.org/10.1590/ S1413-78522013000400001.

18. Urquhart DM, Edwards ER, Graves SE, Williamson OD, Mcneil JJ, Kossmann T, et al. Characterisation of orthopaedic trauma admitted to adult level 1 trauma centres. Injury. 2006;37(2):120-7. PMid:16414050. http://dx.doi.org/10.1016/j.injury.2005.10.016.

19. Jergesen H, Oloruntob D, Aluede E, Grova M, Phillips J, Caldwell A. Analysis of outpatient trauma referrals in a sub-Saharan African orthopedic center. World J Surg. 2011;35(5):956-61. PMid:21360308. http://dx.doi. org/10.1007/s00268-011-1001-2.

20. Gawryszewski VP, Coelho HMM, Scarpelini S, Zan J, Jorge MHPM, Rodrigues EMS. Perfil dos atendimentos a acidentes de transporte terrestre por serviços de emergência em São Paulo, 2005. Rev Saude Publica. 2009;43(2):275-82. PMid:19287873. http://dx.doi.org/10.1590/ S0034-89102009000200008.

21. Reiniger LO, Sousa RMC, Nogueira LS, Costa ALS. Victims of traffic occurrence submitted to surgery procedures: characteristics and perioperative complications. Rev Esc Enferm USP. 2012;46(Esp):58-64. PMid:23250259. http://dx.doi.org/10.1590/S0080-62342012000700009.

22. Franciozi CES, Tamaoki MJS, Araújo EFA, Dobashi ET, Utumi CE, Pinto JA. Epidemiology, treatment and economical aspects multiple of trauma in children and adolescents in a public hospital. Acta Ortop Bras. 2008;16(5):261-5.

23. Deslandes SF, Silva CMFP, Ugá MAD. O custo do atendimento emergencial às vítimas de violências em dois hospitais do Rio de Janeiro. Cad Saude Publica. 1998;14(2):287-99. PMid:9658214. http://dx.doi.org/10.1590/ S0102-311X1998000200013

24. Jorge MHPM, Koizumi MS. Gastos governamentais do SUS com internações hospitalares por causas externas: análise no Estado de São Paulo, 2000. Rev Bras Epidemiol. 2004;7(2) http://dx.doi.org/10.1590/ S1415-790X2004000200012.

25. Batista FS, Silveira LO, Castillo JAQ, Pontes JE, Villalobos LDC. Epidemiological profile of extremity fractures in victims of motorcycle accidents. Acta Ortop Bras. 2015;23(1):43-6. PMid:26327795. http:// dx.doi.org/10.1590/1413-78522015230100998.

26. Thomas V, Sridhar L. Epidemiologic profile of road traffic accident (RTA) cases admitted in a tertiary care hospital: a retrospective study in Hyderabad, Andhra Pradesh. Int J Med Pharm Sci. 2013;03:30-6.

27. Gorios C, Armond JE, Rodrigues CL, Pernambuco H, Iporre RO, Souz PC. Analysis of hospitalization occurred due to motorcycles accidents in São Paulo city. Acta Ortop Bras. 2015;23(4):212-4. PMid:26327804. http://dx.doi. org/10.1590/1413-78522015230400999.

28. Koca K, Ege T, Kurklu M, Ekinci S, Bilgic S. Spiral-medial butterfly fractures (AO-12-B1) in distal diaphysis of humerus with rotational forces: preliminar results of open reduction and plate-screw fixation. Eur Rev Med Pharmacol Sci. 2015;19(23):4494-7. PMid:26698243.

29. Andrade LM, Lima MA, Silva CHC, Caetan JÁ. Acidentes de motocicleta: características das vítimas e dos acidentes em hospital de Fortaleza-CE, Brasil. Rev. Rene. 2009;10(4):52-9.

30. Demirhan R, Onan B, Oz K, Halezeroglu S. Comprehensive analysis of 4205 patients with chest trauma: a 10-year experience. Interact Cardiovasc Thorac Surg. 2009;9(3):450-3. PMid:19541693. http://dx.doi.org/10.1510/ icvts.2009.206599.

31. Consunji RJ, Larona AJL, Jumangit AC, Ameratunga SN. Motorcycle-related trauma in the Philippines resulting in hospital admission: the epidemiology of cases at the Philippine General Hospital. Acta Med Philipp. 2013;47(3):242-5.

32. Ganne N. Estudo sobre acidentes de trânsito envolvendo motocicletas na Cidade de Corumbá e região, Estado do Mato Grosso do Sul, Brasil, no ano de 2007. Rev Pan-Amaz Saude. 2010;1(3):19-24. http://dx.doi.org/10.5123/ S2176-62232010000300003.

33. Katsaragakis S, Drimousis PG, Kleidi ES, Toutouzas K, Lapidakis E, Papadakis $\mathrm{G}$, et al. Interfacility transfers in a non-trauma system setting: an assessment of the Greek reality. Scand J Trauma Resusc Emerg Med. 2010;16:18-14. PMid:20233409.

Recebido em: Maio 12, 2016 Aprovado em: Nov. 22, 2016 